\title{
Management of Femoral Neck Stress Fracture in an Athlete Using Clodronic Acid: a Clinical Case Report
}

\author{
Matteo Vitali ${ }^{1} \cdot$ Pierluigi Pironti $^{1}$ (D) Andreas Drossinos ${ }^{1} \cdot$ Elisa Pesce $^{2} \cdot$ Vincenzo Salini $^{1}$
}

Accepted: 20 September 2019 /Published online: 28 October 2019

(C) The Author(s) 2019

\begin{abstract}
Stress fractures are common injuries caused by repetitive micro-traumas of the bone. Nowadays, they are widespread amongst the athletes and their treatment is mostly based on the limitation of physical activity, the application of ice, and the administration of analgesics and anti-inflammatory drugs. We present a case of a 26-year-old woman who reported a mono-cortical stress fracture in the anatomical neck portion of the right femur treated with Clody ${ }^{\circledR} 200 \mathrm{mg} / 4 \mathrm{ml}$. The patient is an amateur runner who complained a progressive pain and functional limitation of the hip joint. No history of recent trauma was present as well as X-rays evidence of fractures. A subsequent magnetic resonance imaging study showed the presence of a mono-cortical stress fracture in the anatomical neck portion of the right femur. The patient was treated with 1 vial of Clody® $200 \mathrm{mg} / 4 \mathrm{ml}$ a day for 7 days, following 1 vial every 15 days for 2 months without any further therapy as well as physical activity limitation, showing a faster clinical and radiological recovery compared with the most of therapies described in the literature. Concerning our positive experience, the purpose of this study is to give a starting point for further research in order to enlarge the number of studies about that specific approach.
\end{abstract}

Keywords Stress fractures $\cdot$ Femoral neck $\cdot$ Athletes $\cdot$ Micro-trauma $\cdot$ Clodronic acid

\section{Introduction}

Stress fractures are common injuries due to repetitive stress on the bone, occurring mostly in lower extremities. They are widespread amongst professional as well as amateur athletes. Indeed, repetition of movements that overload the bone tissue can lead to the development of micro-traumas, which add up to generate the fracture [1].

Pain, tenderness, and focal edema of the affected site represent the typical clinical presentation [2]. The first radiological assessment should be done through a plane X-ray, followed by further investigation, such as magnetic resonance imaging (MRI), to better assess the lesion [2,3]. Treatment is mostly based on conservative strategies including limitation of

This article is part of the Topical Collection on Medicine

Pierluigi Pironti

pierluigi.pironti@libero.it

1 Department of Orthopedics and Traumatology, San Raffaele Scientific Institute, Via Olgettina 60, 20132 Milan, MI, Italy

2 Medicine and Surgery, Vita-Salute San Raffaele University, Milan, Italy physical activities, application of ice, analgesics, and nonsteroidal anti-inflammatory drugs (NSAID) administration [3]. Recovery time depends on the severity of the lesion. The Arendt and Griffiths (A\&G) classification is based on radiological parameters aiming to define the length of recovery time. In particular, fractures are divided into four stages depending on the severity: grade 1 results to be less severe and consequently needs 3 weeks of recovery, grade 2 needs from 3 to 6 weeks for cure, grade 3 needs from 12 to 16 weeks, and for grade 4 , at least 16 weeks for recovery are generally necessary [3].

We present here a case of a 26-years-old healthy woman who reported a grade 4 mono-cortical stress fracture in the anatomical neck portion of the right femur due to fatigue, treated with Clody® $200 \mathrm{mg} / 4 \mathrm{ml}$. Following the suggested treatment, the resting period needed was 9 weeks instead of 16 weeks, as expected by the $A \& G$ classification [3].

\section{Case Description}

The patient is an amateur runner that reported a progressive pain that gradually increased during the 3 weeks prior to her 
first visit to the general practitioner (July 2018). She complained of weight-bearing pain, as well as difficulties walking the stairs, and tenderness around the right hip. Range of motion was not compromised; however, she experienced pain during both active and passive movement of the femoroacetabular joint along all axes (intra rotation, extra rotation, flexion, extension, abduction, and adduction). No neurovascular deficit was present. Since no history of recent or past trauma was reported and the ultrasound and X-rays initially prescribed were negative, a conservative oral NSAID therapy was administered. As the pain continued to increase, the patient was examined a couple of weeks later and an MRI was prescribed to further investigate the cause of distress (August 2018). The MRI confirmed a grade 4 A\&G stress fracture of the right femoral anatomical neck (Fig. 1).

Stress fractures are injuries involving individuals who usually perform repetitive and intense movements such as dancers, soldiers, and professional athletes. Two types of stress fractures are commonly documented: insufficiency fractures and fatigue fractures. The former occur in a mechanically compromised bone structure with normal loading forces [3]. On the other side, fatigue fractures occur in physiological bone stimulated by excessive mechanical loads, involving $20 \%$ of all sport injuries [4].

Essentially, stress fractures are the result of a succession of micro-traumas that over time lead to gradual weakening of the bone architecture. Indeed, repetitive movements that exceed physiological bone elasticity could damage the tissue, leading to osteocyte apoptosis and activation of osteoclastic differentiation mediators such as the receptor activator of nuclear factor kappa-B ligand (RANKL) [1]. The activation of the aforementioned mechanism is fundamental in bone remodeling, and consequently in microlesion repair. However, an increase in the porosity of the bone tissue can occur, with consequent weakening of the whole bone structure [1].

Since repetition of the noxious gesture is the determining factor for the development of the pathology, a timely diagnosis is essential to avoid a potentially compromising deterioration. Patients often report pain of the affected site which increases during activity [2]. An increase in the intensity of training schemes is commonly a trigger for the onset of the fracture. For this reason, it is fundamental to investigate a possible variation in the sports habits of the patient. As in our case, during the initial phases of the pathology, the X-rays can be negative having a sensitivity of $10 \%$ which grows to $30 \%$ only after 3 weeks. Therefore, a MRI is a necessary to assess the patient, as recommended by the American College of Radiology whose sensitivity is $100 \%$ and specificity $85 \%$ [2].

Risks associated with the development of a stress fracture can be both intrinsic and extrinsic [3]. Extrinsic factors are linked to training conditions, such as playground (rigid or unstable), inadequate equipment, particularly intense training programs, and eating habits (calcium and vitamin D deficiencies are particularly associated with bone disorders) [5]. Intrinsic risks, on the other hand, are mainly determined by anatomical variation and sex. Women are more prone to develop stress fractures, especially in case of menstrual disorders, with a consequent increase in the risk of stress fracture by 2 or 4 times [4].

Treatment must begin as soon as possible in order to avoid any further deterioration [3]. The various extrinsic risks must be eliminated. In particular, changes in the equipment used, changes in the training program, and verification of sufficient calcium and vitamin D intake should be appropriate [6].

Concerning the regeneration of the fracture, the patient is generally instructed to limit his or her physical activities, use ice, rest, and use crutches to avoid loading weight on the injured limb. Physiotherapy is also recommended in order to reduce the extent of muscular hypotrophy and prevent loss of articular range of motion of the limb involved. Eventually, analgesic drugs such as NSAIDs can be administered to reduce pain [3]. However, it has been noted that NSAIDs may interact with the regeneration of the fracture since these drugs block the cyclooxygenases that are fundamental in skeletal
Fig. 1 MRI showing a grade 4 $A \& G$ stress fracture of the right femoral anatomical neck

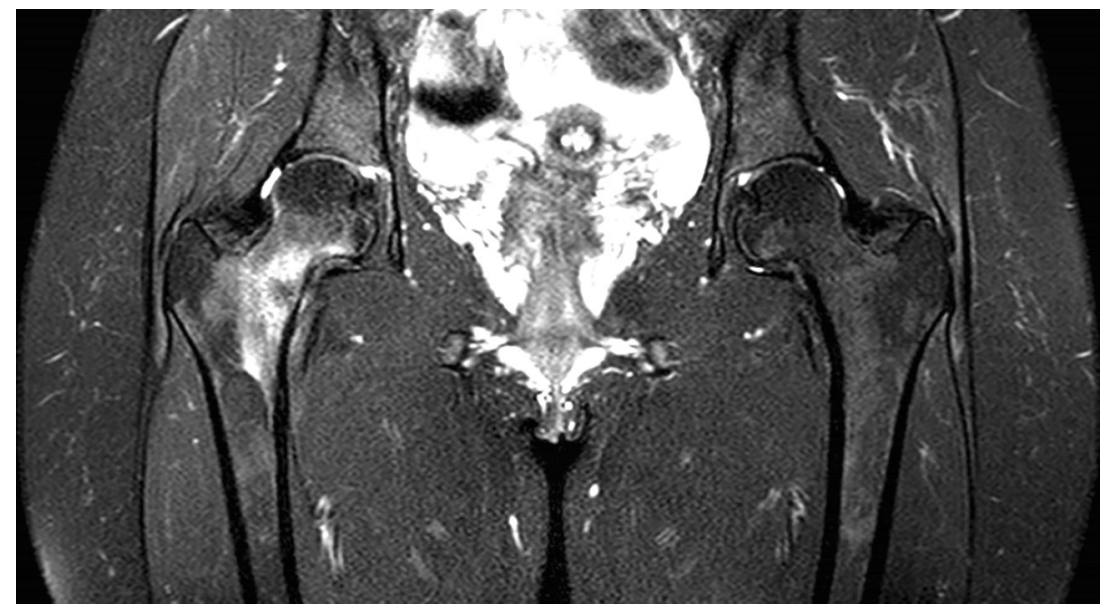


Fig. 2 MRI showing correct bone regeneration and a complete recovery of physiological anatomy

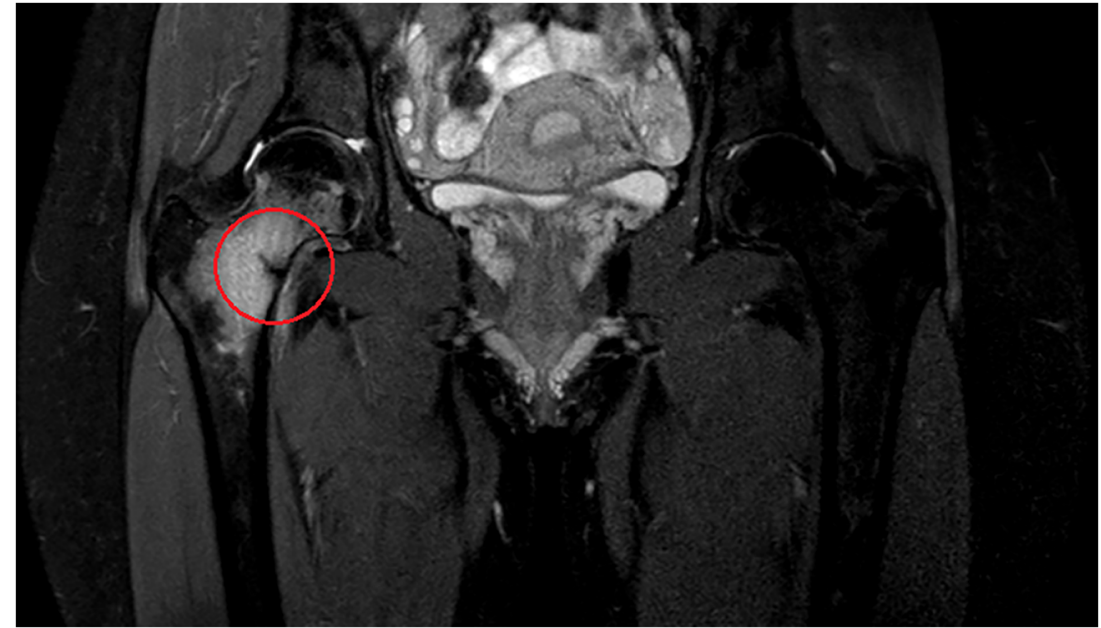

repair. Studies conducted by Xinping Zhang et al. show how cyclooxygenase-2 (COX-2) -/- mice have difficulties in the formation of the bone callus in tibial fractures with longer healing times [7].

Further conservative approaches have been described in the literature. Numerous studies have proposed the possibility of short-term bisphosphonates administration in the treatment of stress fractures; nevertheless, their role in the bone healing remains still unclear. However, several reports suggest the existence of bisphosphonates fracture repair properties, probably due to a stabilizing action on the bone callus [8]. Moreover, physical therapies may play an important role in the stress fractures management. In particular, extracorporeal shock wave therapy (ESWT) has shown positive results due to their angiogenetic properties [8, 9].

In our case, during the first days of September following the MRI results, the patient began a treatment consisting in Clody® $200 \mathrm{mg} / 4 \mathrm{ml}$. We administered one vial a day for 7 days, following one vial every 15 days for the next 2 months. No further therapies were prescribed as well as crutches physiotherapy and instrumental therapy. At the end of the prescribed treatment an additional MRI was requested to access the status of the fracture.

\section{Conclusion}

During the first weeks of treatment the pain increased significantly. Nevertheless, at 2 months of follow-up, the patient was able to return to daily and athletic physical activities, showing a normal and pain-free articular range of motion and good stability of the joint. No adverse conditions were reported by the patient during the pharmacological treatment. The MRI confirmed correct bone regeneration and a complete recovery of physiological anatomy (Fig. 2).

According to the A\&G classification, the treatment we proposed provides much shorter recovery times and also does not include the use of crutches, physiotherapy, and antiinflammatory drugs.

Concerning our positive experience and the poor literature about this approach, we think it could be useful to better analyze its effectiveness through a double-blinded clinical trial. In light of the demanding enrollment of that specific category of patients, the aim of our study is to arouse curiosity of the researchers in order to create new opportunities to further explore this field.

Authors' Contributions MV performed the therapy, EP and PP authored the article, and $\mathrm{AD}$ and VS provided the final review. All authors read and approved the final manuscript.

\section{Compliance with Ethical Standards}

Conflict of Interests The authors declare that they have no conflict of interests.

Ethics Approval and Consent to Participate This study was conducted following the principles of the Declaration of Helsinki and with the patient's permission expressed through a written consent.

Consent for Publication A consent for publication was obtained prior to the article writing.

Open Access This article is distributed under the terms of the Creative Commons Attribution 4.0 International License (http:// creativecommons.org/licenses/by/4.0/), which permits unrestricted use, distribution, and reproduction in any medium, provided you give appropriate credit to the original author(s) and the source, provide a link to the Creative Commons license, and indicate if changes were made.

\section{References}

1. Saunier J, Chapurlat R. Stress fracture in athletes. Jt Bone Spine. 2018;85(3):307-10.

2. Patel DS, Roth M, Kapil N. Stress fractures: diagnosis, treatment, and prevention. Am Fam Physician. 2011;83(1):39-46. 
3. Astur DC, Zanatta F, Gonc G, Moraes ER, Pochini ADC, Ejnisman B. Stress fractures: definition, diagnosis and treatment. Rev Bras Ortop. 2015;1(1):3-10.

4. Moreira CA, Bilezikian JP. Stress fractures: concepts and therapeutics. J Clin Endocrinol Metab. 2017;102(2):525-34

5. Lappe J, Cullen D, Haynatzki G, Recker R, Ahlf R, Thompson K. Calcium and vitamin D supplementation decreases incidence of stress fractures in female navy recruits. J Bone Clin Res. 2008;23(5):741-9.

6. Ross AC, Manson JE, Abrams SA, et al. The 2011 Report on dietary reference intakes for calcium and vitamin D from the Institute of Medicine: what clinicians need to know. J Clin Endocrinol Metab. 2011;96:53-8.
7. Zhang X, Schwarz EM, Young DA, Puzas JE, Rosier RN, Keefe RJO. Cyclooxygenase-2 regulates mesenchymal cell differentiation into the osteoblast lineage and is critically involved in bone repair. $\mathrm{J}$ Clin Invest. 2002;109(11):1405-15.

8. Shima Y, Engebretsen L, Iwasa J, Kitaoka K, Tomita K. Use of bisphosphonates for the treatment of stress fractures in athletes. Knee Surg Sports Traumatol Arthrosc. 2009;17(5):542-50.

9. Leal C, Agostino CD, Gomez S, Fernandez A. Current concepts of shockwave therapy in stress fractures. Int J Surg. 2015;24:195-200.

Publisher's Note Springer Nature remains neutral with regard to jurisdictional claims in published maps and institutional affiliations. 